\title{
MASTER
}

\section{INFORMATION THEORETIC ANALYSIS \\ OF GAMMA-RAY MULTIPLICITIES IN \\ DEEP INELASTIC COLLISIONS}

\author{
by \\ R.D. Levine and S.G. Steadman
}

Department of Physical Chemistry and Institute for Advanced Studies The Hebrew University Jerusalem, Israel
Laboratory for Nuclear Science and Department of Physics, Massachusetts Institute of Technology Cambridge, Mass. 02139, USA

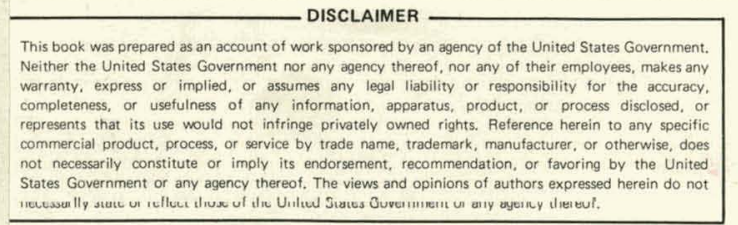




\section{DISCLAIMER}

This report was prepared as an account of work sponsored by an agency of the United States Government. Neither the United States Government nor any agency Thereof, nor any of their employees, makes any warranty, express or implied, or assumes any legal liability or responsibility for the accuracy, completeness, or usefulness of any information, apparatus, product, or process disclosed, or represents that its use would not infringe privately owned rights. Reference herein to any specific commercial product, process, or service by trade name, trademark, manufacturer, or otherwise does not necessarily constitute or imply its endorsement, recommendation, or favoring by the United States Government or any agency thereof. The views and opinions of authors expressed herein do not necessarily state or reflect those of the United States Government or any agency thereof. 


\section{DISCLAIMER}

Portions of this document may be illegible in electronic image products. Images are produced from the best available original document. 
A constrained phase space approach accounts for the magnitude and the trends of the recently observed [1] variance of gamma-ray multiplicity distributions in deep inelastic collisions. 
Measurements of the first and second moments of gamma-räy multiplicity distributions for deeply inelastic collisions have recently been reported $[1,2]$. While the value of the mean multiplicity can be understood [1-4] on the basis of tangential friction between the nuclei $[5,6]$, the magnitude of the variance was posed as a 'severe challenge' [1]. We report here the results of a constrained phase space [7-9] approach which accounts for the observed values of the variance. Given the mean value, $\langle M\rangle$, of the multiplicity we infer the entire angular momentum distribution and, in particular, conclude that the variance is a function of $\langle M\rangle$. The predicted dependence reproduces the trends noted in the experimental data $[1,2]$ : the variance, $v$, is comparable to the mean multiplicity for low values of $\langle M>$ and increases to about half the mean multiplicity for higher values of $\langle M\rangle$. These trends are essentially independent of angle or of the charges of the ejectiles $[1,2]$. An accurate representation of the measured exit energy distributions in heavy-ion induced reactions has previously [10] been achieved by the constrained phase space ('maximal entropy') approach: The present results provide additional demonstration of the usefulness of such an analysis for reactions which cannot be well described in purely: statistical terms. Indeed, for molecular collisions this type of analysis has been successfully applied to very direct processes $[8,9,11]$.

A purely statistical distribution of final states in a reaction is one where all final quantum states (allowed by the conservation laws) are equally probable. Such a distribution is of maximal entropy and is proportional to the density of states. In a direct reaction there may be constraints that preclude a uniform population of the accessible final phase space. The actual distribution $P$ may thus deviate from the distribution $P^{0}$ (the normalized density of states) computed on the assumption that all final quantum states are equiprobable. The constraints that cause such deviance can be identified on dynamical grounds $[7-11]$. The tangential friction model $[5,6]$ suggests that the 
mean value $\langle j(j+1)\rangle$, where $j$ is the angular momentum of the nucleus is an appropriate constraint for sticking collisions. We thus search for the most statistical (maximal entropy) distribution of $j$, which is constrained to yield the specified value of $\langle j(j+1)\rangle$. The result is

$$
P(j)=P^{o}(j) \exp \left[-\dot{\lambda} j(j+1)-\lambda_{0}\right] \text {. }
$$

Here $\lambda_{0}$ is determined by the condition that $P(j)$ is a normalized distribution (and hence is a function of $\lambda$ ). The value of $\lambda$ is determined by the condition that the average value of $j(j+1)$ computed using the distribution (1) agrees with the given mean value. All other averages computed using the distribution (1), and, in particular, $\langle j\rangle$ are thus functions only of $\lambda$ or, equivalently, of $\langle j(j+1)\rangle$. Once the $\lambda$ dependence of $\langle j\rangle$ has been determined one can regard $\lambda$ and hence all other averages as functions of $\langle j\rangle$. For example, if $p^{\circ}(j) \propto(2 j+1)$, then in the limit of high $\langle j\rangle,\langle j\rangle=(\pi / 4 \lambda)^{1 / 2}$, and the variance $v$, defined as usual by $v^{2}=\langle j(j+1)\rangle-\langle j\rangle^{2}$ is given by

$$
v=\langle j\rangle[(4-\pi) / \pi]^{1 / 2} \simeq 0.52\langle j\rangle \text {. }
$$

We shall argue below that (2) is the 'saturation dependence' [1] of the observed variance on the mean multiplicity.

In the region of higher internal energies typical of the products of deep inelastic collisions one expects the nuclear level density to be roughly that of a degenerate Fermi gas [12]. For a nucleus of rotational state $j$ its dominant dependence on $j$ is of the form

$$
\rho(j, U) \propto(2 j+1) \exp \left[2(a U)^{1 / 2}\right]=(2 j+1) \exp \left[-E_{r o t} /\left(E^{\star} / a\right)^{1 / 2}\right] \rho(0, U) .
$$

Here $U=E_{\text {rot }}+E^{*}$ where $E^{*}$ is the excitation energy (with pairing corrections) and $\mathrm{E}_{\text {rot }}$ is the rotational energy. $a$ is the level density parameter. In the actual computation we have used the rigid body estimate $E_{\text {rot }}=\hbar^{2} j(j+1) / 2 I$ and have taken $a=A / 8 \mathrm{MeV},[12]$.

For deep inelastic collisions the nuclear 'temperature' parameter $\left(E^{*} / a\right)^{1 / 2}$ 
typically exceeds $1 \mathrm{MeV}$ whereas the rotational energy is often less. At low j's (and high internal excitations) the density of states is thus dominated by the $(2 j+1)$ term: Hence, in the limit of large negative $Q$ values the saturation behavior, eq. (2), should result. $\left(E^{\star}=Q_{g g}-Q\right.$, where $Q_{g g}$ is the ground state to ground state $Q$ value and $Q$ is the difference between the final and initial kinetic energies in the center-of-mass system.) At higher $Q$ values one should use $P^{O}(j) \propto:(j, U)$ and combining (1) and (3)

$$
P(j)=(2 j+1) \exp \left[-\beta j(j+1)-\lambda_{0}\right] \text {. }
$$

Here $\beta$ is a composite parameter, $\beta=\lambda+\hbar^{2} / 2 I\left(E^{*} / a\right)^{1 / 2}$. In general, we expect the second term in $\beta$ to be important (and $\beta$ to be larger) in the region of high $Q$ values. The orbit matching condition implies that for channels close to the incident channel the average rotational energy will be small and thus $\lambda$ [defined in eq. (1)] is 1arge.

Using the angular momentum distribution (4) we have computed the variance as a function of the mean multiplicity $<M>$. For the purpose of comparing the calculated $\langle j\rangle$ values to the experimental data we have assumed (see, e.g. [4]) that $2\langle M\rangle=\left\langle j_{1}\right\rangle+\left\langle j_{2}\right\rangle$, where 1 and 2 refer to the two ejectiles. The results are shown vs. $B$ in figure 1 . At high values of $B$ (high $Q$ or channels near the projectile charge) the mean multiplicity is low and the variance is of comparable value to the mean. As $B$ decreases (negative Q's or channels away from the incident one) $\langle M\rangle$ increases and the variance settles at about half the mean multiplicity as is observed experimentally $[1,2]$. For all values of $\beta$ the skewness $s,\left(s=\left\langle(M-\langle M\rangle)^{3}\right\rangle / v^{3}\right)$ is small but posjtive (roughly $s \approx 0.6$ independently of $\langle M\rangle$ ). The preliminary experimental results [1] are however that at the larger $\langle M\rangle$ values $s$ is small but negative. At the larger $\langle M>$ values the range of final $j$ 's allowed by the distribution (1) is considerable and may therefore be restricted by the range of 
total angular momentum available in a deep inelastic collision. If one introduces such a cutoff on the distribution (4), the skewness does indeed become small and negative at the higher $<M>$ values. One should also note that the observed value of $\langle M\rangle$ is determined by the angular momentum distributions of both ejectiles. The results shown in figure 1 were obtained on the assumption that both distributions have a common value of $\beta$. Indeed, on maximal entropy grounds, this is the correct result if the only constraint is on the combined mean values, i.e., on $\left\langle M^{2}\right\rangle$. Alternatively, one can use various friction models (e.g., [13]) to partition the angular momentum between the two nuclei. The magnitude of the parameter $\beta$ for the two nuclei is then no longer the same. However, for all reasonable models we find that the dependence of the variance on $\langle M>$ remains essentially that shown in the figure. Since only the value of the skewness summed over final $Z^{\prime}$ 's was reported [1], the experimental results could not be used to distinguish between the different models.

The angular momentum distribution for the ejectiles from a deep inelastic collision was determined as one of maximal entropy subject to a constraint on the mean rotational energy (i.e., on $\langle j(j+1)\rangle$ ). The constraint can be interpreted in terms of tangential friction. The computed magnitude of the variance and its trend as $\langle M\rangle$ varies conforms to the available experimental results $[1,2]$. A complete validation of the results and the proper interpretation of the magnitude of the skewness must await the experimental determination of the skewness for specific exit channels and, ultimately, of the entire multiplicity distribution.

The work of S. G. S. is performed under Contract No.DE-ACO2-76ER03069 of the U. S. Department of Energy. 
The work of R. D. L. was supported by the U. S. Air Force Office of Scientific Research, Grant AFOSR77-3135. The present collaboration was supported by a Young Faculty Career Development Grant provided by the General Electric Corporation (S. G. S.) and the Institute of Advanced Studies of the Hebrew. University of Jerusalem.

\section{References}

[1] P. R. Christensen et al.; Phys. Rev. Letts. 40 (1978) 1245.

[2] G. R. Young et al., Phys. Rev., to be published.

[3] M. M. Aleonard et a1., Phys. Rev. Letts. 40 (1978) 622; P. Glyssel et al., Phys. Rev. Letts. 38 (1977) 33.

[4] R. Albrecht et al., Phys. Rev. Letts. 34.(1975) 1400.

[5] J. P. Bondorf, Nuclear Spectroscopy and Nuclear Reactions with Heavy Ions, Int'1 School of Physics 'Enrico Fermi' (Varena, 1974).

[6] C. F. Tsang, Phys. Scr. 10 (1974) 90; J. Galin, J. de Phys. 11 (1976) C5-83.

[7] Y. Alhassid and R. D. Levine, Phys. Rev. A18 (1978) 89; Phys. Rev. C. 20 (1979) 1775.

[8] R. D. Levine and R. B. Bernstein in Dynamics of Molecular Collisions (P1enum, New York, 1977), Part B.

[9] R. D. Levine et al., Chem. Phys. Letts. 19 (1973) 1.

[10] R. D. Levine et al., Phys: Rev. Letts. 47 (1978) 1537.

[11] R. D. Levine, Ann. Rev. Phys: Chcm. 29 (1978) 59.

[12] A. Bohr and B. R. Mottelson, Nuclear Structure, (Benjamin, N.Y., 1969), $\checkmark 1 \mathrm{p} 155$.

[13] T. M. Cormier et al., Phys. Rev. Al6 (1977) 215. 


\section{Legend}

The mean (continuous curve) and variance (broken curve) of the gamma ray multiplicity distribution vs. $-10^{-6} / \beta^{2}$. $\beta$ is the dimensionless parameter introduced in equation 4 . In the limit where $\left\langle M^{2}\right\rangle$ is large, $\left\langle M^{2}\right\rangle \propto \beta^{-1}$. The scale of the abscissa was chosen to mimic roughly a $Q$ scale $\left(E^{*}=Q_{g g}-Q \propto(B-\lambda)^{-2}\right.$, cf. the discussion of equation 4$)$. As is observed [1,2] saturation [cf. eq. (2)] sets in for large negative $Q$ values, whereas $v$ and $\left\langle M>\right.$ are about comparable for $Q \approx Q_{g g}$. 


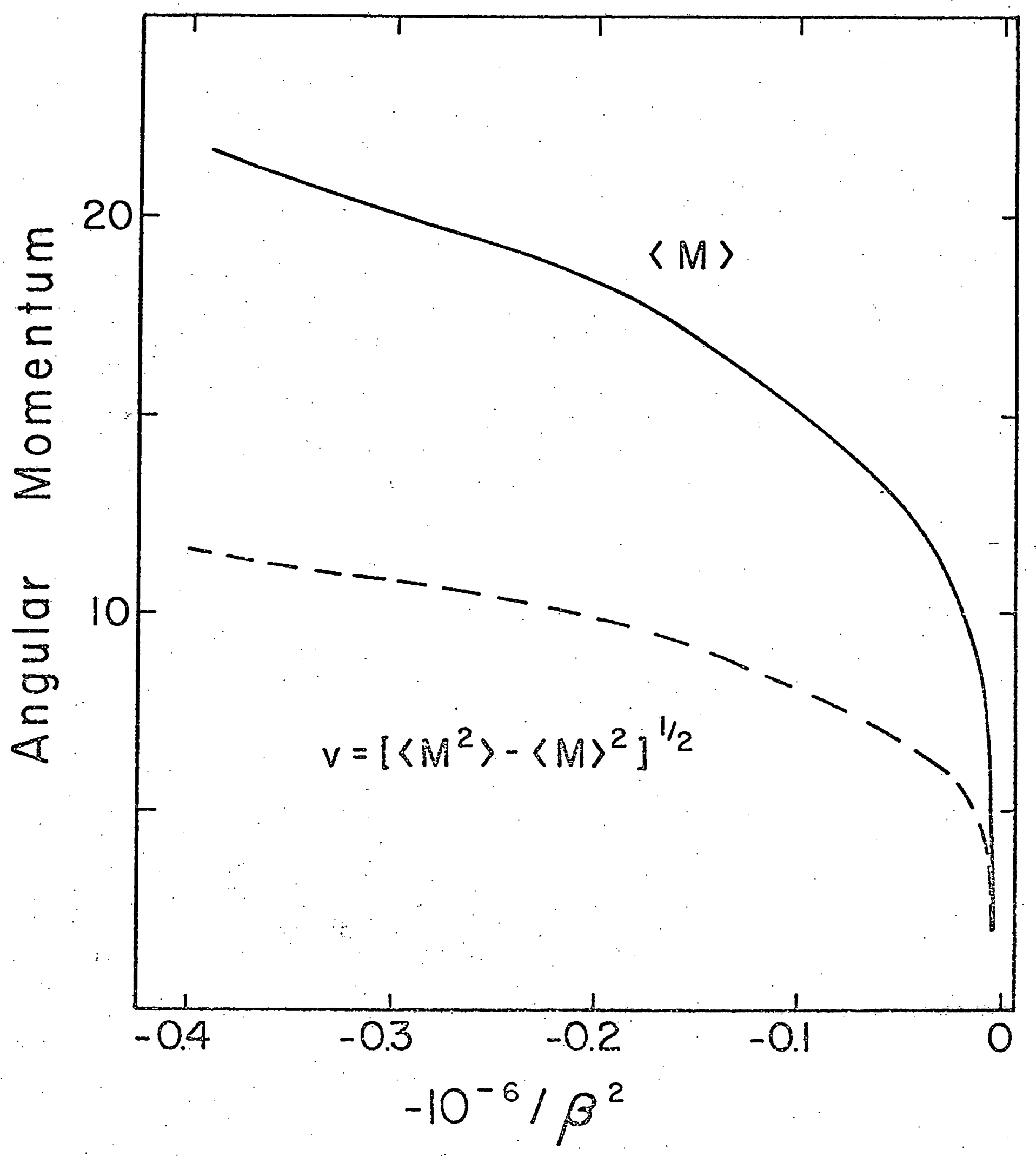

